\title{
Scaling relations of branching pulsatile flows
}

\author{
Carla Silva ${ }^{\text {a, b }}$, A. Heitor Reis ${ }^{b, c, *}$ \\ a Polytechnic Institute of Tomar, Quinta do Contador, 2300-313 Tomar, Portugal \\ b Évora Geophysics Centre, Rua Romão Ramalho, 59, 7000-671 Évora, Portugal \\ c Physics Department, University of Évora, Rua Romão Ramalho, 59, 7000-671 Évora, Portugal
}

\section{A R T I C L E I N F O}

\section{Article history:}

Received 13 May 2013

Received in revised form

16 September 2014

Accepted 18 September 2014

Available online

\section{Keywords:}

Branching flows

Scaling

Murray's law

Pulsatile flow

Constructal theory

\begin{abstract}
A B S T R A C T
Apparently complex flow structures obey to scaling relations that enable to make it viable the study of their configuration and flow dynamics. This is the case of flow structures that exhibit several branching levels and are thought to perform optimally.

Here we present scaling relations of diameters and lengths of branching cylindrical channels with pulsatile flows, and compare them with other relations published in the literature. It is shown that, under constant global volume of the flow tree, and for zero pulse frequency these scaling relations reduce to Murrays's law of consecutive diameters. Optimal scaling depends on pulse frequency, distensibility of the channel walls, and asymmetry of the daughter vessels. In case that in addition to global volume of the flow tree, the pressure head is also kept constant, a similar scaling law of channel lengths emerges that holds together with the law of diameter scaling. The effect of channel distensibility is shown to be somehow important, such that for achieving optimal performance (lowest impedance) channels with lower relative distensibility must have their diameter increased. Results are compared with those of other models for the case of some arteries.
\end{abstract}

(c) 2014 Elsevier Masson SAS. All rights reserved.

\section{Introduction}

Murray's Law [1-3] which states that the "cube of the radius of a parent vessel equals the sum of the cubes of the radii of the daughters" stays as a landmark scaling law of geometries of branching channels with non-turbulent flows (see Fig. 1). It was originally proposed by Cecil D. Murray (1926) for the circulatory and respiratory systems, yet later on has been proved to hold for every branching laminar flow [3,4].

Murray stated in his original work [2] that physiologic organization should be based on principle and pointed out minimum work and balanced cooperation of the organs in the body as the best candidate for such a principle. Sherman [3] provided a full derivation of Murray's law based on that principle. Allometric scaling laws are common in biology and, with the purpose of their explanation, approaches have been developed based on optimal performance of the whole system, either trough minimization of energy dissipation [5] or through flow configuration that enables

\footnotetext{
* Corresponding author. Physics Department, University of Évora, Rua Romão Ramalho, 59, 7000-671 Évora, Portugal.

E-mail addresses: carlasilva@ipt.pt (C. Silva), ahr@uevora.pt, ahrgoo@gmail.com (A.H. Reis).
}

maximum flow access [6]. West and co-workers [5] presented a general model of allometric scaling relations (WBE model) in that the ratio between the diameters of consecutive arteries, $D_{k+1} / D_{k}$, is $n^{-1 / 2}$ for arteries, and $n^{-1 / 3}$ for small vessels ( $n$ stands for branching ratio), regardless of the length of the vessels.

Murray's Law has also been considered in the context of engineered systems. About a decade ago, Bejan and coworkers [4] proved that Murray's law may be deduced from a general principle - the Constructal Law (1997) - which states: "For a finite-size system to persist in time (to live), it must evolve in such a way that it provides easier access to the imposed currents that flow through it." (see for instance Ref. [7]). Said another way, Constructal Law entails evolution of flow architecture in such a way that under the existing constraints the distribution of flow resistances evolves in time to achieve minimum global flow resistance.

Under the conjecture that Nature has optimized in time the living structures, Reis and coworkers [8] applied both Murray's Law and Constructal Law to successfully anticipate some architectural features of the lung tree. More complete information about the successful application of the Constructal Law may be found in Bejan [6], Reis [9], and Bejan and Lorente [10].

However, we note that with respect to optimal performance Mauroy et al. [11] have put forward the idea that "the optimal system is dangerously sensitive to fluctuations or physiological 


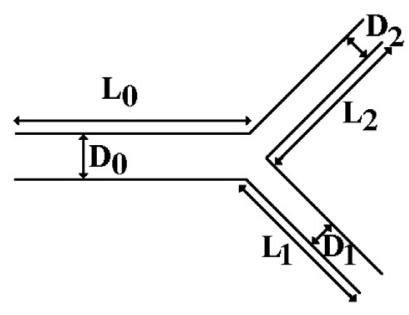

Fig. 1. Branching channels with distensible walls ( $D$ - diameter; $L$ - length).

variability, such that physical optimality cannot be the only criterion for design".

With respect to optimal scaling in asymmetric branching, Bejan [7] has shown that

$\frac{D_{1}}{D_{0}}=\left(1+\xi^{3}\right)^{-1 / 3}, \frac{D_{1}}{D_{2}}=\frac{L_{1}}{L_{2}}$

where $D_{2} / D_{1}=L_{2} / L_{1}=\xi$, is the asymmetry factor of daughter vessels, and the subscripts $0,1,2$ represent the parent and each one of the daughter vessels, respectively. The Eq. (1) which relates homothety coefficient with asymmetry factor further adds to the study of scaling in asymmetric flows, which are shown to be important for achieving optimal performance of flow trees [12].

In the following we will further extend this analysis to find out the scaling relations of branching pulsatile flows.

\section{Pulsatile flows}

Flows that develop in circulatory trees are ubiquitous in Nature. In some animals, namely the vertebrates, blood is rhythmically pumped through the entire body at a broad range of pulse rates. It is recognized that pulsatile flow performs best than continuous flow because it induces lower overall resistances [13] and also better blood perfusion [14].

The most complete model of pulsatile flows, was put forward by Womersley [15] who solved Navier-Stokes equation in channel with elastic walls and periodic pressure forcing, and provided formulas for the pressure wave, and the radial and longitudinal components of the velocity field in the arteries. This work that stays as a landmark in the field was used as one of the basis of the WBE model [5].

Since then, other works have appeared that modeled pulsating flows in rigid channels [16]. Noteworthy are those of Nield and Kuznetzov [17], Siegel and Perlmutter [18] and Faghri et al. [19], albeit these studies were also carried out under the "rigid channel" assumption. Models using analogy with electric circuits date back to about several years ago. Remarkable by its complexity are those of Tsitlik et al. [20], Avolio [21], or recently that of Mirzaee et al. [22].

With the purpose of optimizing branching structures with pulsatile flows, in what follows we will further explore the parallel RC model. Though Womersley's equations describe pulsatile flows accurately, they are quite complex, and not easy to handle analytically in the study of branching vessels. This is why we use an RC model as a suitable description of pulsatile flow. In this model the flow induced by the pressure wave "charges the capacitor" (the arterial elastic walls) while it is braked by a "Poiseuille resistance" in the flow direction. The rationale for using Poiseuille flow, rather than considering a more complex model based on the Navier-Stokes equation is explained in the following.
Let us start from Navier-Stokes equation for unidirectional flow: $\partial u / \partial t+u \cdot \operatorname{grad} u=-\rho^{-1} \operatorname{grad} P+\nu$ lap $u$. In the case of pulsatile flow in arteries, the inertial terms may be discarded because they are, at least, of one order of magnitude smaller than the other terms, as it is shown through scale analysis. In this way, let $u$ denote average blood velocity, $\tau$ characteristic time related to pulse wave frequency, $L_{c}$ the characteristic length in the flow direction, $D$ vessel diameter, $\rho$ blood density, $\Delta P$ pressure variation along the vessel and $\nu$ blood kinematic viscosity. Then, by assuming the following scale values for large arteries: $u \sim 10^{-1} \mathrm{~ms}^{-1}, \tau \sim 1 \mathrm{~s}, L_{c} \sim 1 \mathrm{~m}, D \sim 10^{-3} \mathrm{~m}$, $\Delta P \sim 10^{3} \mathrm{~Pa}$ and $\nu \sim 10^{-5} \mathrm{~m}^{2} \mathrm{~s}^{-1}$, the orders of magnitude (in $\mathrm{ms}^{-2}$ ) of the terms in the Navier-Stokes equation are: $\partial u / \partial t \sim 10^{-1}$, $u$.grad $u \sim 10^{-2}, \rho^{-1} \operatorname{grad} P \sim 1, \rho^{-1}$ lap $u \sim 1$, therefore justifying the use of Poiseuille flow as a first approach in the study of the human arterial system. Models that include the term $\partial u / \partial t$ lead to greater complexity in the calculations but did not cause a change in the conclusions. For example, the RLC model developed by Jager and co-workers [23] accounts for the "sleeve effect", which arises from the interaction between viscous and inertial terms in the Navier-Stokes equation. However, in the same study [23] it was shown that the "sleeve effect" is important in some arteries at frequencies higher than $15 \mathrm{rad} \mathrm{s}^{-1}$, which is somehow beyond the normal range of the human pulse frequency.

In real systems, pressure waves of some frequency travel all along the circulatory trees. Energy travels in the form of enthalpy plus mechanical energy of the bulk fluid, and in the form of elastic energy of the vessel walls.

As the basis for building up a model of a pulsatile flow driven by a pressure difference $\Delta P$ in a vessel of length $L$ and diameter $D$, one starts from the Hagen-Poiseuille equation in the form:

$I=k_{A}^{-1} L^{-1} D^{4} \Delta P$,

where $I$ is current $\left(\mathrm{m}^{3} \mathrm{~s}^{-1}\right), k_{A}=128 \mu \pi^{-1}$, in which $\mu$ is dynamic viscosity of the fluid. In pulsatile flow, both $\Delta P$ and $D$ are functions of time, and therefore the same happens with the conductance $K_{p}=k_{A}^{-1} L^{-1} D^{4}$. In what follows the variables $D, L, V$ standing for geometric features of vessels with pulsatile flow represent values averaged over a cycle. In this way, as a first approximation we will consider the actual conductance in the channel as the sum of the average conductance (corresponding to diameter $D$ ) plus the deviation corresponding to diameter variation with pressure, i.e.

$K_{p}(t)=K+\bar{K}^{\prime}=k_{A}^{-1} L^{-1} D^{4}\left(1+2 \beta(\mathrm{d} P / \mathrm{d} t)_{0} \Delta t\right)$,

where $\beta=(2 / D)(\mathrm{d} D / \mathrm{d} P)$ is the distensiblity coefficient and $\Delta t$ is the time elapsed after the channel diameter has reached the average value. The Eq. (3) shows that the conductance is the sum of two terms: the first one corresponds to the inverse of the usual resistance while the second one is equivalent to the inverse of a capacitive resistance. This aspect is made clearer if we consider $I(t)=K_{p}(t) \Delta P(t)$ together with Eq. (3) to obtain:

$I \approx k_{A}^{-1} L^{-1} D^{4} \Delta P+2 k_{A}^{-1} L^{-1} D^{4}(\Delta t) \beta \Delta P(\mathrm{~d} P / \mathrm{d} t)_{0}$,

Eq. (4) shows that the flow in a channel with elastic walls is composed of two terms: one corresponds to a resistive current,

$I_{r}=k_{A}^{-1} L^{-1} D^{4} \Delta P$,

while the other matches up a capacitive current,

$I_{C}=2 I_{r}(\Delta t) \beta(\mathrm{d} P / \mathrm{d} t)_{0}$.

with capacitance $C=2 I_{r}(\Delta t) \beta$, (see Fig. 2). 


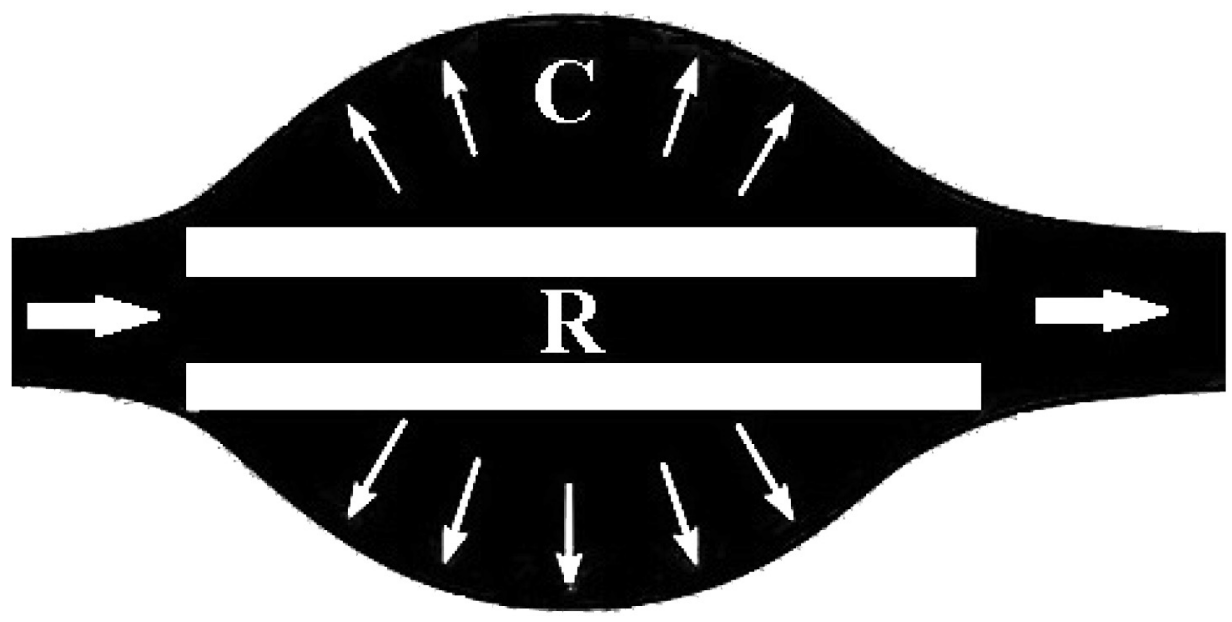

Fig. 2. Channel wall distensibility is accounted for by an analog to electric capacitance, while flow resistance is that of Hagen-Poiseuille flow.

As the global conductance is the sum of the respective conductances, one concludes that an equivalent parallel RC circuit is the model suitable for describing pulsatile flow (see Fig. 3).

\section{Minimization of impedance in branching pulsatile flows}

As pressure increases in the channel, the elastic walls are strained to accommodate more fluid. The capacitance $C$, which has the same meaning as compliance in vessel physiology, is defined as $C=\mathrm{d} V / \mathrm{d} P$. For a cylindrical channel, with volume $V=\pi D^{2} L / 4$, one obtains:

$C=k_{B} D^{2} L$

with

$k_{B}=\pi \beta / 4$.

The Eq. (7) provides the opportunity for conferring some significance to $\Delta t$ in Eq. (6). Then, by putting together $C=2 I_{r}(\Delta t) \beta$ and $C=k_{B} D^{2} L$ we get $\Delta t=\left(\pi D^{2} L / 8\right) / I_{r}$. Therefore, $\Delta t$ would stand for the time required for the averaged current $I_{r}$ to fill half of the channel volume. However, we note that Eq. (3) was put forward simply with the purpose of justifying the existence a capacitive flow, together with a resistive flow in the case of a deformable channel, and will not be used in what follows.

Additionally, the resistance is given by (see Eq. (5)):

$R=k_{A} L D^{-4}$.

Let us consider a channel with elastic walls with a pressure wave of frequency $\omega$. By analogy with the electric circuit model (Fig. 3), the total impedance $Z$ of such channel reads:

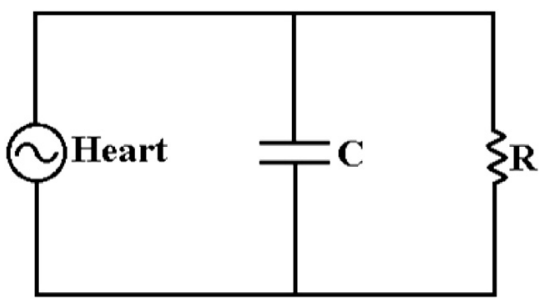

Fig. 3. Pulsatile flow as an analog to a parallel RC circuit.
$|Z|=R\left(1+\omega^{2} R^{2} C^{2}\right)^{-1 / 2}$

which with Eqs. (7) and (8) and $x=L / D, y=D^{3}, k=k_{A} k_{B}$ becomes:

$|Z|=k_{A} x y^{-1}\left(1+\omega^{2} k^{2} x^{4}\right)^{-1 / 2}, \quad k x^{2}=1 / \widehat{\omega}$

where $\widehat{\omega}$ represents the characteristic frequency of the channel.

\subsection{Minimization of global impedance under constant volume}

Now, let us consider a channel that branches in two different channels, as represented in Fig. 1. For the vast majority of flow systems in the conditions described above (Eqs. (7) and (8)) we have (see Appendix).

$|Z|_{T}=|Z|_{o}+\left(|Z|_{1}^{-1}+|Z|_{2}^{-1}\right)^{-1}$.

By taking into account Eq. (10), the Eq. (11) reads.

$$
\begin{aligned}
|Z|_{T}= & \frac{k_{A} x_{0} y_{0}^{-1}}{\left(1+\omega^{2} k_{0}^{2} x_{0}^{4}\right)^{1 / 2}} \\
& +\left(\frac{\left(1+\omega^{2} k_{1}^{2} x_{1}^{4}\right)^{1 / 2}}{k_{A} x_{1} y_{1}^{-1}}+\frac{\left(1+\omega^{2} k_{2}^{2} x_{2}^{4}\right)^{1 / 2}}{k_{A} x_{2} y_{2}^{-1}}\right)^{-1} .
\end{aligned}
$$

The volume of a cylindrical vessel is $V=(\pi / 4) D^{2} L$. With $\tilde{V}=4 V / \pi$, the total volume $\tilde{V}_{T}$ occupied by the flow system is.

$$
\tilde{V}_{T}=D_{0}^{2} L_{0}+D_{1}^{2} L_{1}+D_{2}^{2} L_{2}=x_{0} y_{0}+x_{1} y_{1}+x_{2} y_{2} \text {. }
$$

In Eqs. (12) and (13) $x$ and $y$ are free variables that describe the branching structure. The condition of easiest flow access is achieved with the minimization of the global flow impedance under constant global volume of the channels, $\tilde{V}_{T}$,

$\mathrm{d}|Z|_{T}-\lambda \mathrm{d} \tilde{V}_{T}=0$

where $\lambda$ is a constant.

We chose the aspect ratios $\left\{x_{0}, x_{1}, x_{2}\right\}$ as design variables subject to optimization for the reason that $x=L / D$ defines channel geometry better than either $D$ or $L$ alone. 
By minimizing the global impedance $|Z|_{T}$ (Eq. (12)) under constant volume with respect to each of design variables $\left\{x_{0}, x_{1}, x_{2}\right\}$ one obtains, respectively:

$\lambda=\frac{k_{A}}{y_{0}^{2}}\left(A_{0}^{-1 / 2}-2 \omega^{2} k_{0}^{2} x_{0}^{4} A_{0}^{-3 / 2}\right)$,

$\lambda=\frac{k_{A} B^{2}}{x_{1}^{2}}\left(A_{1}^{1 / 2}-2 \omega^{2} k_{1}^{2} x_{1}^{4} A_{1}^{-1 / 2}\right)$,

$\lambda=\frac{k_{A} B^{2}}{x_{2}^{2}}\left(A_{2}^{1 / 2}-2 \omega^{2} k_{2}^{2} x_{2}^{4} A_{2}^{-1 / 2}\right)$.

where $A_{i}=1+\omega^{2} k_{i}^{2} x_{i}^{4}, \quad i=0,1,2, \quad$ and $\quad B=\left(A_{1}^{1 / 2} x_{1}^{-1} y_{1}+\right.$ $\left.A_{2}^{1 / 2} x_{2}^{-1} y_{2}\right)^{-1}$. Therefore, with the definition of $A_{i}$, and Eqs. (16) and (17) one obtains:

$\left(A_{1}^{1 / 2}-2 A_{1}^{-1 / 2}\right)\left(A_{1}-1\right)^{-1 / 2}=\left(A_{2}^{1 / 2}-2 A_{2}^{-1 / 2}\right)\left(A_{2}-1\right)^{-1 / 2}$.

A solution to Eq. (18) is.

$A_{1}=A_{2}$.

This first result, indicates that for minimal resistance to flow the characteristic frequencies $\widehat{\omega}_{i}=1 / R_{i} C_{i}=1 / k_{i} x_{i}^{2}$ (see Eq. (10)) of the two daughter channels must be equal.

By using $A_{i}=1+\omega^{2} k_{i}^{2} x_{i}^{4}$ together with Eqs. (16), (17) and (19) one obtains:

$\frac{D_{1}}{D_{0}}=\theta^{1 / 6}\left(1+\xi^{3}\right)^{-1 / 3}$,

$\frac{D_{2}}{D_{0}}=\theta^{1 / 6}\left(1+\xi^{-3}\right)^{-1 / 3}$

where

$\theta=\left(2 A_{i}^{-3 / 2}-A_{i}^{-1 / 2}\right) /\left(2 A_{0}^{-3 / 2}-A_{0}^{-1 / 2}\right), i=1,2$

and $\xi=L_{2} / L_{1}$ stands for branching asymmetry. Taking together the Eqs. (20) and (21) one has,

$D_{0}^{3}=\theta^{-1 / 2}\left(D_{1}^{3}+D_{2}^{3}\right)$.

For non-pulsatile flow $\left(A_{i}=1 ; \theta=1\right)$ the Eq. (23) reduces to the known form of Murray's Law:

$D_{0}^{3}=D_{1}^{3}+D_{2}^{3}$

Additionally, from the Eqs. (20) and (21) we obtain the following relationship:

$\frac{D_{2}}{D_{1}}=\frac{L_{2}}{L_{1}}=\xi$,

or, in view of Eq. (19):

$x_{1}=x_{2} ; \beta_{1}=\beta_{2}$.

The Eq. (26) show that in optimal flow branching the daughter channels share the same aspect ratio $x$ and distensibility coefficient $\beta$.
By using the Eqs. (19)-(21), and (26) as the results of the optimization, and $\widehat{\omega}_{i}=1 / k_{i} x_{i}^{2}$, and $A_{i}=1+\omega^{2} k_{i}^{2} x_{i}^{4}$, we obtain the minimal impedance from Eq. (12) in the form.

$|Z|_{T, \mathrm{opt}}=\frac{4}{\pi \beta_{0} L_{0} D_{0}^{2} \widehat{\omega}_{0} A_{0}^{1 / 2}}\left(1+\frac{L_{1}}{L_{o}}\left(1+\xi^{3}\right)^{1 / 3} \phi^{-1}\right)$,

where

$\phi=\theta^{2 / 3}\left(\frac{A_{1}}{A_{0}}\right)^{1 / 2}$.

Now we are able to assess the effect of pulsation on the impedance of the bifurcation represented in Fig. 1. By noting that non-pulsatile flow corresponds to $A_{1}=A_{0}=1$ and $\theta=1$, therefore $|Z|_{T}$ reduces to the usual resistance $R$, we can represent the ratio $|Z|_{T} / R$ as a function of frequency as shown in Fig. 4 for various values of $\chi=\widehat{\omega}_{1} / \widehat{\omega}_{0}$, and for the particular case of symmetric branching $\xi=1$ and $L_{1}=0.5 L_{0}$. For the same $x=L / D$, we note that $\chi<1$ stands for a daughter channel of higher distensibility with relation to the father channel $\left(\beta_{1} / \beta_{0}>1\right)$, while $\chi>1$ represents just the reverse. As shown in Fig. 4 the effect of pulsation only becomes significant as the pulse frequency approaches the characteristic vessel frequency $\widehat{\omega}_{0}$, namely for $\omega / \widehat{\omega}_{0}>0.1$. In the case when the daughter channel is less distensible $(\chi>1)$ pulse frequency significantly reduces flow impedance, an effect that for the same $x=L / D$ increases with the inverse of the distensibility ratio $\beta_{0} / \beta_{1}=\widehat{\omega}_{1} / \widehat{\omega}_{0}=\chi$ (see Fig. 4). On the contrary, in the case when the daughter channel is more distensible pulse frequency increases impedance as the pulse frequency falls in the vicinity of the characteristic parent channel frequency $\widehat{\omega}_{0}$.

\subsection{Minimization of global impedance under constant volume and} pressure head

Reis [9] has shown that minimal global flow resistance in a branching tree under constant total pressure head $\Delta P$, is achieved with a tree configuration in which total flow resistances allocated

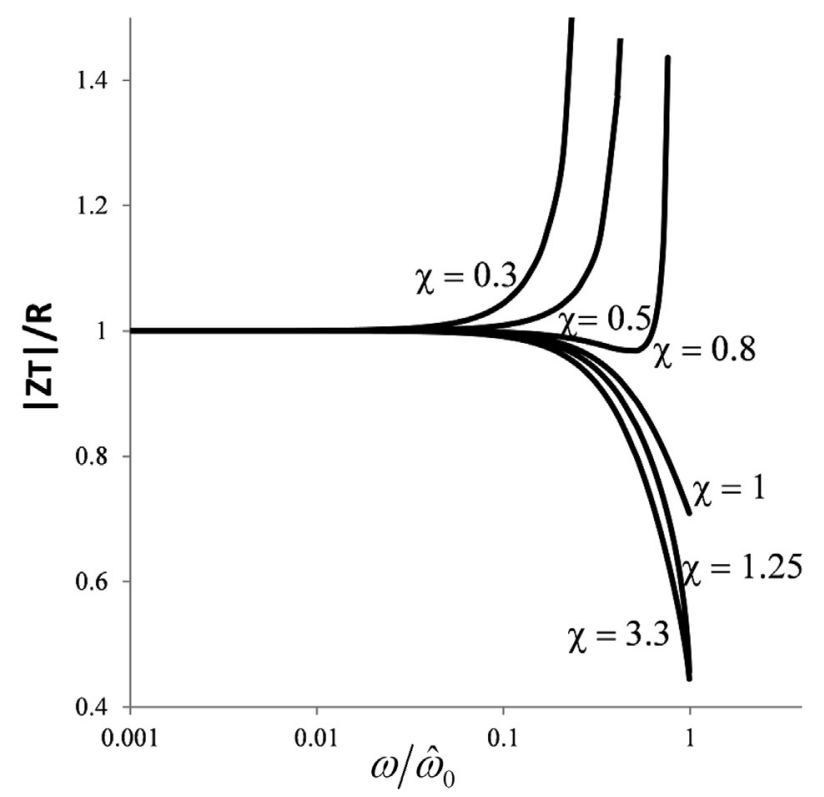

Fig. 4. The effect of the relative characteristic frequencies of parent $\left(\widehat{\omega}_{0}\right)$ and daughter channel $\left(\widehat{\omega}_{1}\right)$, (with $\left.\chi=\widehat{\omega}_{1} / \widehat{\omega}_{0}=0.8,0.5,0.3,1,1.25,3.3\right)$, on impedance as function of pulse frequency. 
to flow are the same at each branching level. This means that in the best performing (optimal) flow system design both the overall resistance and pressure drop distribute in such a way that their respective values do not change from a branching level to the next one. This result enables generalize the scaling laws of channel length for pulsatile flow. The Eq. (27) shows that the minimal impedance of the branching channel is the sum of 2 terms: the first one corresponds to the father channel while the second one stands for the global impedance of the branching channels. Because they must have the same value one obtains:

$\frac{L_{1}}{L_{0}}=\phi\left(1+\xi^{3}\right)^{-1 / 3}$,

where we used $A_{i}$ as defined above. Similarly, one obtains:

$\frac{L_{2}}{L_{0}}=\phi\left(1+\xi^{-3}\right)^{-1 / 3}$,

To conclude, in case that both volume and pressure head are kept constant, the global impedance of an optimal tree with $N-1$ branching levels with pulsatile flow of frequency $\omega$ reads,

$|Z|_{T, \mathrm{opt}}=N \frac{4}{\pi \beta_{0} L_{0} D_{0}^{2} \widehat{\omega}_{0} A_{0}^{1 / 2}}$,

where $\widehat{\omega}_{0}=\left(k_{0} L_{0}^{2} / D_{0}^{2}\right)^{-1}$ is the characteristic frequency of the parent channel.

\subsection{Scaling of unstrained channel diameters with different distensibilities}

We recall that $D$ in Eqs. (20)-(25) refer to channel diameters averaged over a cycle. Because average channel diameter $D$ may be expressed in terms of its unstrained value $D_{\text {us }}$ plus a term corresponding to an average dilation, i.e. $D=D_{\mathrm{us}}\left(1+\beta P^{+} / 2\right)$, where $P^{+}=P(D)-P\left(D_{\text {us }}\right)$, the Eqs. (20) and (21) read:

$\frac{D_{1 \mathrm{us}}}{D_{\text {0us }}}=\frac{\left(1+\beta_{0} P^{+} / 2\right)}{\left(1+\beta_{1} P^{+} / 2\right)} \theta^{1 / 6}\left(1+\xi^{3}\right)^{-1 / 3}$,
$\frac{D_{2 \mathrm{us}}}{D_{\text {Ous }}}=\frac{\left(1+\beta_{0} P^{+} / 2\right)}{\left(1+\beta_{2} P^{+} / 2\right)} \theta^{1 / 6}\left(1+\xi^{-3}\right)^{-1 / 3}$.

As a consequence the unstrained diameters ratios of parent to daughter channels will be affected as function of channel distensibilities and the pressure excursion during the pulse (see Eq. (32)).

Fig. 5 shows the unstrained diameter ratios $\left(D_{1 \mathrm{us}} / D_{0 u s}\right)$ as function of frequency for the case when $\chi=1.25$, and for various values of $\beta_{0} P^{+}$. With $\chi$ fixed, because $\chi=\widehat{\omega}_{1} / \widehat{\omega}_{0}=\left(\beta_{0} / \beta_{1}\right)\left(x_{0}^{2} / x_{1}^{2}\right)$ (see Eqs. (7)-(10)), note that the ratio $\beta_{0} / \beta_{1}$ depends only upon the ratio $x_{0}^{2} / x_{1}^{2}$, therefore making it possible to explore both the cases when $\beta_{1}>\beta_{0}$ and $\beta_{1}<\beta_{0}$. We observe that for optimal performance (lowest impedance) the unstrained diameter ratios must decrease with $\beta_{0} P^{+}$, which means that decreasing distensibility of parent channel leads to increase in its diameter. The same behavior is observed for the case when $\chi=0.8$ (see Fig. 6). Specifically for $\omega / \widehat{\omega}_{0}>0.1$ the unstrained diameter ratios $\left(D_{1 \text { us }} / D_{0 \text { us }}\right)$ increase with pulse frequency when $\chi=1.25$, and decrease with pulse frequency when $\chi=0.8$.

The human arterial system is an example of a tree with pulsatile flow in which the average pressure head varies in time. Therefore, optimal scaling of such a tree is described by the Eqs. (19)-(28) and (32).

A key aspect of scaling that match minimal impedance in branching channels with pulsatile flow, and with variable pressure

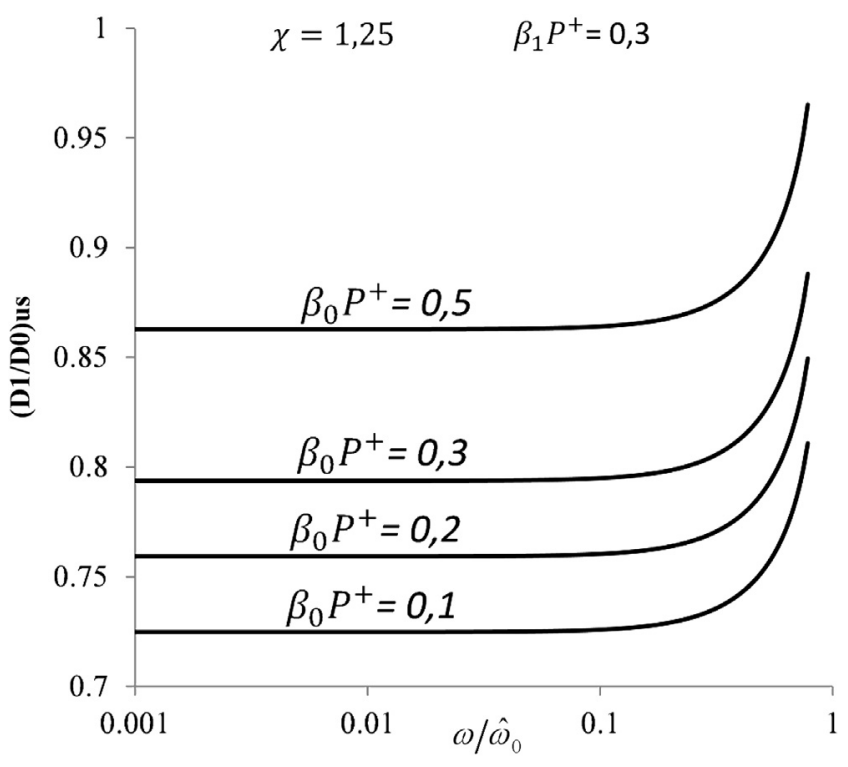

Fig. 5. Daughter to parent unstrained diameter ratios as function of pulse frequency for various channel distensibilities for the case when $\chi=1.25$.

head, is that scaling depends upon pulse frequency. For nonpulsatile flow $\left(\omega=0, A_{i}=1\right)$ in channels with asymmetric branching the Eqs. (20) and (21) reduce to the already known scaling for continuous flow (Eq. (1)).

The Eqs. (20)-(23) and (29)-(30) represent a generalization of Murray's Law for pulsatile flow in a channel with branching asymmetry. Asymmetry of branching is accounted for the parameter $\xi=L_{2} / L_{1}$, which through Eqs. (20) and (21) assigns a smaller diameter to the shorter branch. On the other hand, the global impedance of the pulsatile flow tree depends upon the pulse frequency $\omega$. This is a new and very important result that will be explored in a subsequent study of the human circulatory system viewed as a flow tree with pulsatile flow.

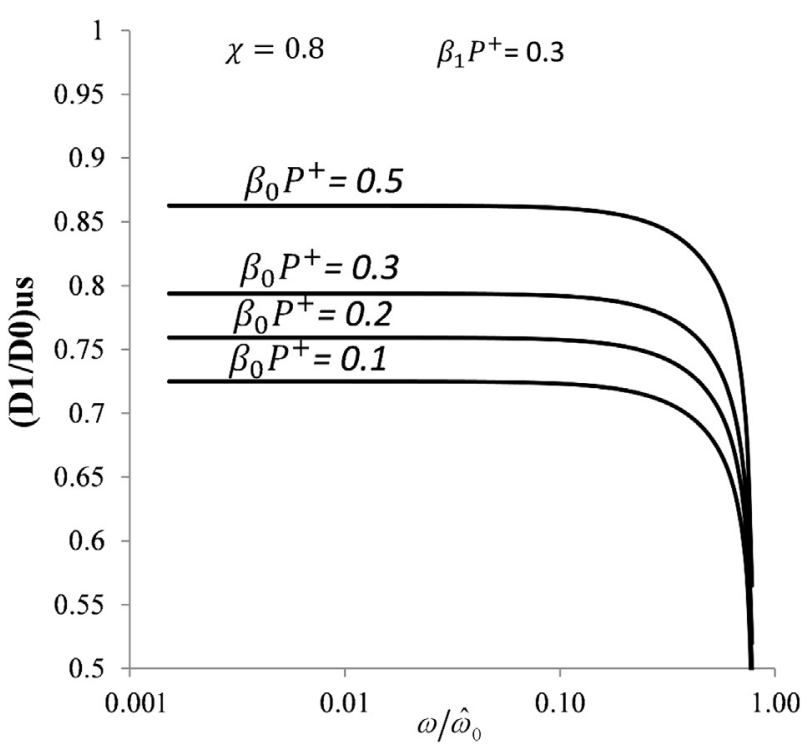

Fig. 6. Daughter to parent unstrained diameter ratios as function of pulse frequency for various channel distensibilities for the case when $\chi=0.8$. 


\subsection{Comparison of the results of the models of Murray, WBE and this model}

In order to compare the predictions of the model above developed with those of other models that present scaling relations for diameters in dichotomous branching, we start from the general scaling relation:

$\frac{D_{n+1}}{D_{n}}=a(1+\varepsilon)^{-\alpha}$,

where as a rule $\varepsilon=1$ appears in the scaling relations of dichotomous branching, $\alpha$ is the scaling exponent, and $a$ is an additional parameter. To the parameters in Eq. (33) Murray's law assigns the fixed values: $a=1, \varepsilon=1$ and $\alpha=1 / 3$, while in the WBE model [5] $a=1, \varepsilon=1$, and $\alpha=1 / 2$ for the branching levels up to a nonspecified level $k$, while $\alpha=1 / 3$ "for large $k$, corresponding to small vessels" [5]. In the present model, $a=\theta^{1 / 6}, \varepsilon=\xi^{3}$ or $\varepsilon=\xi^{-3}$ (depending on the degree of asymmetry of daughter vessels), and $\alpha=1 / 3$ (see Eqs. (20) and (21)). For the sake of comparison we further define the variable

$\Psi=\ln \left(\frac{D_{n+1}}{D_{n}}\right)$,

Therefore, Murray's law is represented by $\Psi=-(1 / 3)$ $\ln 2=0.231$, in the WBE model $\Psi=-(1 / 2) \ln 2=0.347$, or for large branching order $\mathrm{k}, \Psi=-(1 / 3) \ln 2=0.231$. In the present model, $\Psi=(1 / 6) \ln \theta-(1 / 3) \ln \left(1+\xi^{ \pm 3}\right)$.

To calculate $\Psi$ we used the extensive dataset of diameters, lengths and distensibilities of arteries provided in Ref. [24]. We kept the identification number of the each arterial segments used in Fig. 2 and Table 2 of Ref. [24]. The ratio of lengths $\xi=L_{2} / L_{1}$ respects to arterial segments that converge at a bifurcation. On the values given in Ref. [24] we have no absolute guarantee that some of these segments do not have small (or very small) intermediate branches.
This can occur especially in very long segments in which it is likely to find small branches not represented in Fig. 2 and Table 2 of Ref. [24]. A sign of this is that the proximal and distal diameters of these segments differ a lot. For this reason, the data for lengths of arterial segments are used with some caution. We chose arterial segments that correspond to dichotomous branching in which parent and daughter vessels are clearly defined. In total 22 arteries were included in the calculation of $\Psi$.

Table 1 presents the $\Psi$ values as predicted by Murray's law, the WBE model, and the present model. For the most part of the cases the present model predicts the observed values with better approximation than Murrays's law, or the WBE model. This is especially true for the cases of asymmetric branching. The cases in which predictions deviate a lot from the observed values respect to very long vessels, i.e. those that are likely to present very small branches not considered in the dataset used.

Based on the present model, a recently published study of the arterial structure [25] also showed that, in general arterial lengths are not optimized with respect to hemodynamic performance, and then an explanation was offered for the elongation of the ascending aorta in healthy people during lifetime. The same study also showed that impedance of the ascending aorta, descending aorta and carotid artery decreases during body growth, therefore suggesting a trend for improvement of hemodynamic performance during that period of life.

\section{Conclusions}

In this study, based on the minimization of global impedance, the scaling relations of lengths and diameters of the parent and daughter channels in a branching channel are generalized to the case of pulsatile flow.

It is shown that in case of constant tree volume, scaling depends both on pulse frequency and branching asymmetry. Another important parameter that influences optimal scaling is the

Table 1

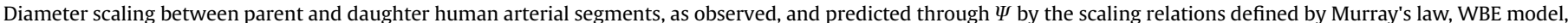
and present model (see Eq. (34)).

\begin{tabular}{|c|c|c|c|c|c|c|c|c|c|}
\hline Artery & $\begin{array}{l}\text { Distensibility } \\
\text { coeff. (Pa s) }\end{array}$ & $D(\mathrm{~mm})$ & $L(\mathrm{~mm})$ & $x$ & $\xi$ & $\begin{array}{l}\Psi \\
\text { Observed }\end{array}$ & $\begin{array}{l}\Psi \\
\text { Murray }\end{array}$ & $\begin{array}{l}\Psi \\
\text { WBE }\end{array}$ & $\begin{array}{l}\Psi \\
\text { This model }\end{array}$ \\
\hline 4 subclavian $\mathrm{A}$ & 2.176 & 9.0 & 34.0 & 3.778 & 0.353 & & & & \\
\hline 7 subclavian $B$ & 1.643 & 8.1 & 422.0 & 52.099 & & -0.105 & -0.231 & -0.347 & -0.016 \\
\hline 6 vertebral & 1.095 & 3.7 & 149.0 & 40.270 & & -0.889 & -0.231 & -0.347 & -1.057 \\
\hline 7 subclavian $B$ & 1.643 & 4.7 & 422.0 & 89.787 & 0.285 & & & & \\
\hline 8 radial & 1.118 & 3.7 & 235.0 & 63.514 & & -0.239 & -0.231 & -0.347 & -0.008 \\
\hline 9 ulnar A & 1.148 & 3.7 & 67.0 & 18.108 & & -0.239 & -0.231 & -0.347 & -1.262 \\
\hline 9 ulnar $\mathrm{A}$ & 1.148 & 3.4 & 67.0 & 19.706 & 2.165 & & & & \\
\hline 10 interosseous & 0.810 & 2.1 & 79.0 & 37.619 & & -0.482 & -0.231 & -0.347 & -0.804 \\
\hline 11 ulnar B & 1.043 & 3.2 & 171.0 & 53.438 & & -0.061 & -0.231 & -0.347 & -0.032 \\
\hline 14 aortic arch 2 & 3.361 & 20.8 & 39.0 & 1.875 & 0.654 & & & & \\
\hline 18 thoracic aorta 1 & 3.196 & 18.9 & 52.0 & 2.751 & & -0.096 & -0.231 & -0.347 & -0.082 \\
\hline 19 subclavian A & 2.108 & 11.0 & 34.0 & 3.091 & & -0.637 & -0.231 & -0.347 & -0.507 \\
\hline 41 abdominal aorta & 2.221 & 10.4 & 20.0 & 1.923 & 1.000 & & & & \\
\hline 42 common iliac & 1.793 & 7.9 & 59.0 & 7.468 & & -0.275 & -0.231 & -0.347 & -0.231 \\
\hline 43 common iliac & 1.793 & 7.9 & 59.0 & 7.468 & & -0.275 & -0.231 & -0.347 & -0.231 \\
\hline 42 common iliac & 1.793 & 7.0 & 59.0 & 8.429 & 0.347 & & & & \\
\hline 50 external iliac & 1.613 & 6.4 & 144.0 & 22.500 & & -0.090 & -0.231 & -0.347 & -0.014 \\
\hline 51 inner iliac & 1.238 & 4.0 & 50.0 & 12.500 & & -0.560 & -0.231 & -0.347 & -1.070 \\
\hline 37 abdominal aorta $\mathrm{C}$ & 2.371 & 11.8 & 20.0 & 1.695 & 3.313 & & & & \\
\hline 38 renal & 1.448 & 5.2 & 32.0 & 6.154 & & -0.819 & -0.231 & -0.347 & -1.207 \\
\hline 39 abdominal aorta D & 2.303 & 11.6 & 106.0 & 9.138 & & -0.017 & -0.231 & -0.347 & -0.009 \\
\hline 50 external iliac & 1.613 & 6.1 & 144.0 & 23.607 & 0.284 & & & & \\
\hline 52 femoral & 1.328 & 5.2 & 443.0 & 85.192 & & -0.160 & -0.231 & -0.347 & -0.008 \\
\hline 53 deep femoral & 1.208 & 4.0 & 126.0 & 31.500 & & -0.422 & -0.231 & -0.347 & -1.266 \\
\hline 52 femoral & 1.328 & 3.8 & 443.0 & 116.579 & 0.936 & & & & \\
\hline 55 anterior tibial & 0.930 & 2.6 & 343.0 & 131.923 & & -0.379 & -0.231 & -0.347 & -0.199 \\
\hline 54 posterior tibial & 1.035 & 3.1 & 321.0 & 103.548 & & -0.204 & -0.231 & -0.347 & -0.265 \\
\hline
\end{tabular}


coefficient of distensibility of the channel walls, which is a parameter widely used in the characterization of the arteries in the circulatory tree.

In the limit of zero pulse frequency these scaling relations match Murray's law of diameters and also the scaling relations of lengths of branching channels with minimal global flow resistance under the existing constraints.

We also show that if the additional constraint of constant global pressure head is imposed to the flow in parent and daughter channels, the optimal ratio of daughter to parent channels lengths follows a law similar to that of channel diameters.

In case that the daughter channel has lower relative distensibility global impedance decreases with pulse frequency. Conversely, if the daughter channel is more distensible pulse frequency increases impedance as the pulse frequency falls in the vicinity of the characteristic frequency of the parent channel.

The effect of the relative distensibility of parent and daughter channels is shown to be important somehow. In this way, the channel with lower relative distensibility must have their diameter increased to perform optimally, i.e. to achieve minimal impedance.

For the case of the human arterial trees, the predictions of the present model were compared with those of Murray's law, and the WBE model, and especially in the cases of asymmetric branching provided a better approximation to the observed values.

\section{Acknowledgment}

The authors acknowledge the funding provided by the CGE, under the contract Pest/OE/CTE/UI0078/2014, with FCT (the Portuguese Science and Technology Foundation).

\section{Appendix}

The impedance is represented by a phase vector (phasor), $\boldsymbol{Z}$. For a channel that branches in two different channels (Fig. 1) the overall impedance reads.

$\boldsymbol{Z}_{T}=\boldsymbol{Z}_{o}+\left(\boldsymbol{Z}_{1}^{-1}+\boldsymbol{Z}_{2}^{-1}\right)^{-1}$

where $Z_{i}=R_{i} /\left(1+j \omega \mathrm{R}_{i} \mathrm{C}_{i}\right), i=0,1,2$, and with $j=\sqrt{-1}$. In polar coordinates, the generic phasor reads:

$Z_{i}=\left|Z_{i}\right|\left(\cos \phi_{i}+j \sin \phi_{i}\right)$,

with $\phi_{i}=\arctan \left(\omega \mathrm{R}_{i} \mathrm{C}_{i}\right)$. For the flow system of Fig. 1, from Eqs. (7) and (8), we get

$\phi_{i}=\arctan (32 \omega x \beta \mu)$,

We assume that in (A2) the imaginary part of $\boldsymbol{Z}_{i}$ may be neglected if $\sin \phi_{i}$ is of order $10^{-2}$. In that case, $\cos \phi_{i} \approx 1$. Then, for a flow system that condition implies.

$\omega x \beta \mu<3.2 \times 10^{-4}$
The inequality (A4) is verified for the vast majority of flow systems. For example, for the human arterial system, $\omega x \beta \mu \approx 10 \cdot 10^{2} \cdot 10^{-5} \cdot 10^{-3} \approx 10^{-5}$.

Therefore, in such conditions, Eq. (A1) reduces to.

$|Z|_{T} \cong|Z|_{o}+\left(|Z|_{1}^{-1}+|Z|_{2}^{-1}\right)^{-1}$

With $\sin \phi_{i}$ of order $10^{-2}$, when the two members of (A5) are set equal, the associated relative error is of order $10^{-4}$.

\section{References}

[1] C.D. Murray, The physiological principle of minimum Work: I. The vascular system and the cost of blood volume, Proc. Natl. Acad. Sci. U. S. A. 12 (3) (1926) 207-214.

[2] C.D. Murray, The physiological principle of minimum Work: II oxygen exchange in capillaries, Proc. Natl. Acad. Sci. U. S. A. 12 (5) (1926) 299-304.

[3] T.F. Sherman, On connecting large vessels to small: the meaning of Murray's Law, J. General Physiology 78 (4) (1981) 431-453.

[4] A. Bejan, L.A.O. Rocha, S. Lorente, T- and Y-shaped constructs of fluid streams, Int. J. Therm. Sci. 39 (2000) 949-960.

[5] G.B. West, J.H. Brown, B.J. Enquist, A general model for the origin of allometric scaling laws in biology, Science 276 (1997) 122-126.

[6] A. Bejan, Shape and Structure, from Engineering to Nature, Cambridge University Press, Cambridge, 2000, pp. 1-314.

[7] A. Bejan, Advanced Engineering Thermodynamics, third ed., Wiley, Hoboken, 2006, pp. 840-841.

[8] A.H. Reis, A.F. Miguel, M. Aydin, Constructal theory of flow architectures of the lungs, Med. Phys. 31 (5) (2004) 1135-1140.

[9] A.H. Reis, Constructal Theory: from engineering to Physics, and how flow systems develop shape and structure, Appl. Mech. Rev. 59 (2006) 269-282.

[10] A. Bejan, S. Lorente, Design with Constructal Theory, Wiley, Hoboken, N. York, 2008, pp. $1-490$

[11] B. Mauroy, M. Filoche, E.R. Weibel, B. Sapoval, An optimal bronchial tree may be dangerous, Nature 427 (2004) 633-636.

[12] M. Florens, B. Sapoval, M. Filoche, Optimal branching asymmetry of hydrodynamic pulsatile trees, Phys. Rev. Lett. 106 (2011) 178104.

[13] C. Mavroudis, To pulse or not to pulse, Ann. Thorac. Surg. 25 (1978) 259-271.

[14] K.M. Taylor, W.H. Bain, K.G. Davidson, M.A. Turner, Comparative clinical study of pulsatile and non-pulsatile perfusion in 350 consecutive patients, Thorax 37 (1982) 324-330.

[15] J.R. Womersley, Oscillatory flow in arteries: the constrained elastic tube as a model of arterial flow and pulse transmission, Phys. Med. Biol. 2 (1957) $178-187$.

[16] D.N. Ku, Blood flow in arteries, Annu. Rev. Fluid Mech. 29 (1997) 399-434.

[17] D.A. Nield, A.V. Kuznetsov, Forced convection with laminar pulsating flow in a channel or tube, Int. J. Therm. Sci. 46 (6) (2007) 551-560.

[18] R. Siegel, M. Perlmutter, Heat transfer for pulsating laminar duct flow, ASME J. Heat Transfer 84 (2) (1962) 111-122.

[19] M. Faghri, K. Javadani, A. Faghri, Heat transfer with laminar pulsating flow in a pipe, Lett. Heat Mass Transfer 6 (4) (1979) 259-270.

[20] J.E. Tsitlik, H.R. Halperin, A.S. Popel, A.A. Shoukas, F.C.P. Yin, N. Westerhof, Modeling the circulation with three-terminal electrical networks containing special non-linear capacitors, Ann. Biomed. Eng. 20 (1992) 595-616.

[21] A.P. Avolio, Multi-branched model of the human arterial system, Med. Biol. Eng. Comput. 18 (1980) 709-718.

[22] M.R. Mirzaee, O. Ghasemalizadeh, B. Firoozabadi, Exact simulating of human arteries using lumped model and probing constriction in femoral and carotid arteries, Am. J. Appl. Sci. 6 (5) (2009) 834-842.

[23] G.N. Jager, N. Westerhof, A. Noordergraaf, Oscillatory flow impedance in electrical analog of arterial system: representation of sleeve effect and nonNewtonian properties of blood, Circ. Res. 16 (1965) 121-133.

[24] P. Reymond, F. Merenda, F. Perren, D. Rüfenacht, N. Stergiopulos, Validation of a one-dimensional model of the systemic arterial tree, Am. J. Physiol. Heart Circ Physiol. 297 (2009) H208-H222.

[25] C. Silva, A.H. Reis, Structure and adaptation of arteries to pulsatile flow - the case of the ascending aorta, Med. Phys. 41 (6) (2014) 063701. 